services for STEMI in the very elderly is likely to increase. Our aim was to establish real life outcome in a large sample of patients aged 85 years and over who received PPCI for acute STEMI.

Methods Data was collected retrospectively on all patients aged 85 years and over who were referred and accepted for PCI at our centre between the years of 2013 and 2018 inclusive. Data on patient demographics, characteristics, door-to-balloon time, left ventricular function and mortality was extracted.

Results 172 patients aged 85 and over were referred and accepted for STEMI treatment with PPCI at our regional Coronary Catheterisation Laboratory over the 6 year period. This represented $4.8 \%$ of all referrals over this time. The oldest patient was 99 years old. The absolute number of cases per year did not show an upward trend over the 6 year study period. 164 individuals went on to have emergency diagnostic coronary angiogram, of which 143 cases proceeded to PCI. PCI was successful in $>95 \%$ of cases with a median door-toballoon time was $26: 00$ minutes. $36 \%$ of all patients post-MI had moderate or severe LVSD on echocardiogram. Table 1 illustrates the demographics and findings of our study.

Median hospital stay for all patients was 7 days (range 1 190). 131 of 164 survived to discharge (79.9\%). $94.6 \%$ of these patients were discharged to their own home, and $5.4 \%$ were discharged to a nursing home. Median survival of all

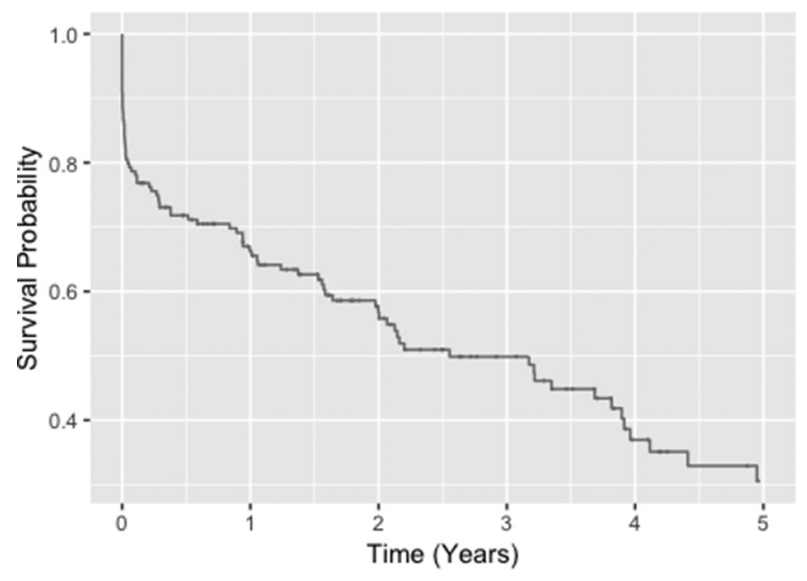

Abstract 64 Figure 1 Survival Post PPCI in 85 and over between $2013-2018$ patients who were discharged was 1.8 years. $55 \%(n=72)$ of discharged patients were deemed functionally independent. When compared to those who mobilised with assistance, functionally independent patients had similar survival rates at 30 days $(98.6 \% \mathrm{v} 96.2 \%)$ but higher survival at 1 year $(90.2 \% \mathrm{v}$ 76.9\%). Table 1 illustrates the demographics and findings of our study. LV function did not appear to influence survival rates.

Patients aged between 85-90 years old (79.3\%) had a higher rate of surviving to discharge (83.1\% v 64.7\%). This group also had better survival rates at 30 days $(83.1 \%$ v $64.7 \%)$ and at one year $(70.8 \%$ v $54.3 \%)$.A Kaplan-Meier survival graph of all patients up to 5 years is shown in figure 1.

Conclusion To our knowledge, this is one of the largest studies reporting real life outcome for PPCI in the very elderly. Despite successful and expedient PPCI in the majority of cases, very elderly patients with STEMI had poor outcome with long hospital stays, and high mortality rates. However, most patients were able to be discharged to their own home. Women, younger patients and functionally independent individuals appear to have slightly more favourable outcomes.

This patient group needs more scrutiny to ascertain whether PPCI should be offered routinely in cases of acute STEMI.

Conflict of Interest nil

\section{NURSE LED RADIAL ACCESS FOR CORONARY ANGIOGRAPHY}

Dorota Wojcik*, Gavin Manmathan, Mahmood Ahmad, Luciano Candilio, Niket Patel, Deven Patel, Gerry Coghlan, Timothy Lockie, Roby Rakhit. Royal Free London NHS Foundation Trust

\subsection{6/heartjnl-2019-BCS.63}

Introduction NHS England announced a new nurse angiographer role with the purpose of reducing hospital waiting lists for elective angiography.

Methods Development of a standardised operating procedure for training in Radial Arterial Access (RAA), guidance on how to minimise the risk and recognise complications and post procedural limb management. Obtaining approval at the new procedures committee. Training program 50 consultant supervised nurse led procedures for (RAA), establishing expertise in

Abstract 64 Table 1 Demonstrating Nurse versus Registrar patient's discomfort score from radial access.

\begin{tabular}{|c|c|c|c|}
\hline & Total & Males & Females \\
\hline No. of patients & 164 & 73 & 91 \\
\hline Mean age (years) & 88.0 & 87.8 & 88.2 \\
\hline Median Stay (days) & 7 & 6 & 7 \\
\hline Proportion of patients with Mod-Severe LVSD post MI & $36.0 \%$ & $43.0 \%$ & $30.8 \%$ \\
\hline Proportion of patients that survived admission & $79.9 \%$ & $79.5 \%$ & $80.2 \%$ \\
\hline Proportion of survivors discharged to home & $94.6 \%$ & $98.3 \%$ & $91.7 \%$ \\
\hline Proportion of survivors discharged to nursing home & $5.4 \%$ & $1.7 \%$ & $8.3 \%$ \\
\hline Proportion of all patients alive at one year & $67.1 \%$ & $64.4 \%$ & $69.2 \%$ \\
\hline
\end{tabular}

Key to Discomfort Score: 0 = no discomfort; 1 = mild discomfort; 2 = moderate discomfort; 3 = severe discomfort 
100 further procedures, followed by roll out of the program to other nursing staff.

Results During training (RAA) cannulation was successful on 1 st attempt in 42 out of 50 (success rate allowing for two attempts 84\%), with no complications recorded. Successful access by a more experienced operator $\mathrm{N}=7$, failed (RAA) $\mathrm{N}=1$. The following 100 were performed independently on unselected patients (success rate $81 \%$; (1st pass 70)), with no complications, need for alternate access routes, $\mathrm{N}=1$.

The following table details the success rate and patient comfort score recorded during 104 procedures performed by a nurse specialist and trainee registrars.

\begin{tabular}{lc}
\multicolumn{1}{l}{ Patient parameters contributing to FRANCE-2 score } & Values \\
\hline & \\
Age $\geq 90$ years & $7.0 \%(n=13)$ \\
BMI $<18.5$ & $1.6 \%(n=3)$ \\
New York Heart Association Class IV & $7.5 \%(n=14)$ \\
Acute pulmonary oedema $\geq 2$ in past year & $6.4 \%(n=12)$ \\
Systolic pulmonary artery pressure $\geq 60 \mathrm{mmHg}$ & $5.9 \%(n=11)$ \\
Critical pre-operative state & $4.3 \%(n=8)$ \\
Respiratory insufficiency & $43.9 \%(n=82)$ \\
Dialysis & $1.1 \%(n=2)$ \\
Transfemoral approach & $93 \%(n=174)$
\end{tabular}

\section{Abstract 65 Table 1}

Conclusion Nurse-led (RAA) is safe and feasible and has the potential to improve catheter laboratory efficiency, patient satisfaction, standardise procedure training and improve staff retention.

Our next steps will be to train more nurses in radial access and progress to nurse led angiography.

Conflict of Interest None

\section{CLINICAL OUTCOMES FOLLOWING ELECTIVE PERCUTANEOUS CORONARY INTERVENTION (PCI) AND SAME DAY DISCHARGE (SDD): A RETROSPECTIVE COHORT STUDY}

${ }^{1}$ Tom Kite*, ${ }^{2}$ Avinash Segaran, ${ }^{3}$ Akhlaque Uddin. ${ }^{1}$ University of Leicester/University Hospitals of Leicester NHS Trust; ${ }^{2}$ Nottingham University Hospitals NHS Trust; ${ }^{3}$ Nottingham university hospitals

\subsection{6/heartjin-2019-BCS.64}

Introduction In 2016, 100,483 patients in the United Kingdom underwent PCI (1). PCI is a guideline recommended treatment for coronary artery disease (2). Contemporary techniques and pharmacotherapy strategies have improved the safety of PCI, thus reducing risk of major adverse cardiovascular events (MACE) and the requirement for overnight stay in elective cases. Published data have demonstrated that the majority of post-procedural complications occur within the initial 6 hours, and that patients can be safely discharged after a period of observation (3). Implementation of these recommendations

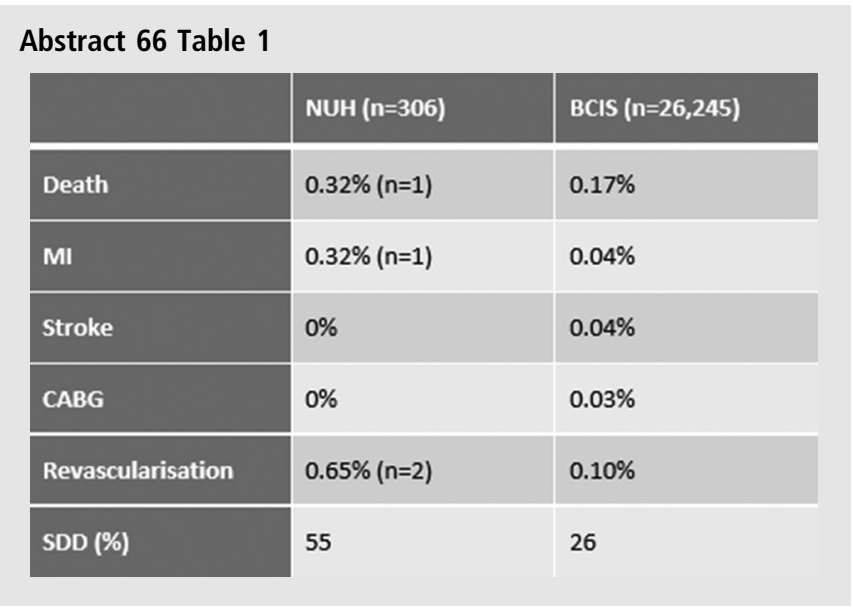

into clinical practice has the potential to provide significant healthcare cost savings.

Objectives This study aimed to evaluate the clinical outcomes of patients undergoing elective PCI at a tertiary cardiac centre, determine rates of elective SDD PCI, and identify potential areas for service improvement.

Methods Elective PCI procedures over a 12-month period (October 2016 - September 2017) were electronically retrieved from our trust's TOMCAT cardiovascular information system. Incidence of MACE at 30 days after PCI was recorded following review of procedure reports, discharge summaries and hospital re-admission data. NHS numbers were used as unique identifiers to ensure robust data collection. Our data was subsequently compared with the British Cardiovascular Intervention Society (BCIS) 2016 audit results.

Results 306 patients underwent elective PCI during the study period. $1.3 \%$ incidence of MACE was recorded (death $0.32 \%$ $[\mathrm{n}=1] ; \quad$ MI $0.32 \% \quad[\mathrm{n}=1]$; urgent revascularisation $0.65 \%$ $[\mathrm{n}=2]$; stroke $[\mathrm{n}=0]$; emergency CABG $[\mathrm{n}=0]) .55 \%(168 /$ 306) of elective PCI was performed as a SDD. 34 patients were admitted overnight, despite undergoing uncomplicated single-vessel PCI. Applying published criteria, SDD would have been appropriate in this group.

Conclusion This study demonstrates that elective PCI at our centre is safe and results in low rates of MACE, with clinical outcomes comparable to national audit data. $55 \%$ of elective PCI at our centre is SDD, considerably higher than BCIS figures. Patients suitable for SDD were identified and will be the focus of future pathway and service improvement.

\section{REFERENCES}

1. British Cardiovascular Intervention Society. BCIS Audit 2016. http://www.bcis.org uk/wp-content/uploads/2018/03/BCIS-Audit-2016-data-ALL-excluding-TAVI-08-032018-for-web.pdf BCIS Audit (Accessed 27th February 2019).

2. Neumann FJ, Sousa-Uva M, Ahlsson A, Alfonso F, Banning AP, Benedetto $U$, et al. 2018 ESC/EACTS Guidelines on myocardial revascularization. Eur Heart J 2019;40(2):87-165.

3. Brayton KM, Patel VG, Stave C, de Lemos JA, Kumbhani DJ. Same-day discharge after percutaneous coronary intervention: a meta-analysis. I Am Coll Cardiol 2013;62(4):275-85.

\section{Conflict of Interest None}

